\title{
High intensity focused ultrasound enhances anti-tumor immunity in melanoma through promoting CD4 Th1 effector T cell responses
}

\author{
HaiYing ZHANG ${ }^{1}$, Kun $\mathrm{HAN}^{2}$,* \\ ${ }^{1}$ Department of Renal Rheumatism, Shanghai Jiaotong University Affiliated Sixth People's Hospital East Campus, NO.222 Huanhu West 3rd Road, Nanhui \\ New Town, Pudong New District, Shanghai 201306, China \\ ${ }^{2}$ Department of Medical Oncology, Shanghai Jiaotong University Affiliated Sixth People's Hospital East Campus, NO.222 Huanhu West 3rd Road, Nanhui \\ New Town, Pudong New District, Shanghai 201306, China
}

*Correspondence: doctor_hankun@163.com https://doi.org/10.37175/stemedicine.v1i4.65

\begin{abstract}
Background: Melanoma accounts for more than $80 \%$ of deaths from all dermatologic cancers, mainly due to its widespread metastasis. High intensity focused ultrasound (HIFU) is a promising technique for cancer therapy. Here, we investigated the efficacy of HIFU against melanoma and the underlying mechanisms.
\end{abstract}

Methods: A melanoma allograft mouse model was established to examine the tumor progression and survival rate. Anti-tumor immunity was determined by measuring cytokines, regulatory T cells (Tregs), Th17 cells and CD8+ effector T cells. Western blot, qPCR, RNAi and luciferase assay were performed to confirm the expression and regulation of microRNA (miR)-9-5p and transforming growth factor beta (TGF- $\beta$ ).

Results: HIFU exposure significantly suppressed melanoma growth and metastasis by activating interferon gamma (IFN- $\gamma$ ) secretion, inhibiting Tregs and Th17 cells, and stimulating CD8+ effector T cells. TGF- $\beta$ was a direct target of miR-9-5p. The anti-tumor effect of HIFU might be mediated through the miR-9-5p/TGF- $\beta$ pathway.

Conclusion: HIFU activates anti-tumor response and alters tumor microenvironment, which may serve as a potential therapeutic strategy for melanoma treatment.

Keywords: Melanoma $\cdot$ HIFU $\cdot$ Antitumor immunity $\cdot$ TGF- $\beta \cdot$ miR-9-5p

\section{Introduction}

Melanoma develops from the melanocytes and is the most malignant type of skin cancer. Although melanoma accounts for only $4 \%$ of skin cancers, it causes more than $80 \%$ of deaths from all dermatologic cancers $(1$, 2). In melanoma patients, the major cause of death is widespread metastasis (3). Melanoma can spread through the lymphatic and/or vascular system to the liver, brain, lung, bone, breast, colon and subcutaneous tissue even at the early stages $(3,4)$. Therefore, investigation and the

Received: July 17, 2020; Accepted: Sep 9, 2020.

() The Author(s). 2020 This is an Open Access article distributed under the terms of the Creative Commons License (http://creativecommons.org/licenses/by/4.0/) which permits unrestricted use, distribution, and reproduction in any medium or format, provided the original work is properly cited. development of effective therapy to inhibit the growth and metastasis have crucial importance in melanoma treatment.

High intensity focused ultrasound (HIFU), also known as focused ultrasound surgery, is a non-invasive therapeutic technique for localized treatment of tumors, which exhibits thermal and mechanical effects: the former one induces cancer cell destruction through coagulation necrosis, while the latter one generates radiation force, cavitation and micro-streaming on tumor tissues $(5,6)$. HIFU has been used to treat various tumors and improve the prognosis of cancer patients (6-11). Uchida et al. reported that the HIFU therapy improved prostate cancer outcomes, and the 10-year survival rate reached $89.6 \%$ among 918 patients (11). Breast cancer is an ideal target for HIFU treatment due to its superficial position. The 
complete ablation rate of HIFU therapy reached up to $71 \%$ in 173 breast cancer patients (10). HIFU treatment also improved the survival outcome of unresectable liver cancer patients, where out of 49 hepatocellular carcinoma (HCC) patients, 39 (79.5\%) had primary effectiveness and only $4(8.1 \%)$ had complications (9). In addition, HIFU exposure was also employed for treatment of kidney cancer, and applied as the palliative treatment for pancreatic cancer $(7,8)$. All these reports have indicated that HIFU is a promising strategy for the treatment of various cancers.

Although HIFU exposure has been used for the treatment of multiple cancers, the underlying molecular mechanisms are not yet well understood. Cumulative evidence has suggested that cell death and tissue damage during HIFU are closely linked to immune response, which is induced by the cell debris after cancer cell destruction, as well as tumor microenvironment alteration such as the changes of T cell subsets (12-14). Xia et al. reported the anti-tumor immune responses of HIFU exposure in HCC mouse model, the authors demonstrated that 14 days of treatment could significantly promote the secretion of interferon gamma (IFN- $\gamma$ ) and tumor necrosis factor alpha (TNF- $\alpha$ ), enhance the cytotoxicity of cytotoxic $\mathrm{T}$ lymphocytes (CTL), and increase the number of CD8+ effector T cells (15). The dendritic cells (DC) were also found activated after HIFU exposure in a colorectal cancer mouse model: there was about 4-fold increase in CD11c+ cells and more than 5-fold CFSE+ DC accumulation in lymph nodes after HIFU exposure, accompanying with enhanced CTL activity and increased IFN- $\gamma$ production (12). However, the therapeutic effect of HIFU exposure on melanoma treatment and the underlying mechanism is poorly defined.

In the current study, we demonstrated that HIFU exposure could significantly suppress melanoma growth and metastasis through activating the anti-tumor immunity and altering the tumor microenvironment in mouse and cell models. We further revealed that HIFU-induced decrease in regulatory T cells (Tregs) and Th17 cells was regulated by the microRNA (miR)-9-5p/transforming growth factor beta (TGF- $\beta$ ) pathway. Notably, HIFU exposure also increased the number of CD8+ effector $\mathrm{T}$ cells in melanoma tissues. This study may pave the way for the clinical application of HIFU in melanoma treatment.

\section{Materials and Methods Animals}

6-8 weeks old C57BL/6J mice were employed in the current study, which were ordered from Shanghai Laboratory Animal Center (China). The mice were housed under standard breading conditions with 12 light/12 dark cycle, at $23^{\circ} \mathrm{C}$ with $40-60 \%$ humidity, standard chow diet feeding, with water accessible at all times. All mouse experiments have been approved by the Shanghai Jiaotong University Affiliated Sixth People's Hospital East Campus.

\section{Cell culture}

The murine skin melanoma cell line B16-F10 (CRL$6475^{\mathrm{TM}}$ ) was acquired from American Type Culture Collection (Manassas, USA). B16-F10 cells were cultured in PRMI-1640 medium (Thermo Fisher Scientific, Waltham, USA) supplemented with 10\% heat-inactivated fetal bovine serum, $2 \mathrm{mM}$ L-glutamine, $100 \mathrm{U} / \mathrm{mL}$ penicillin and $100 \mu \mathrm{g} / \mathrm{mL}$ streptomycin. Cells were placed in $5 \% \mathrm{CO} 2$ cell culture incubator at $37^{\circ} \mathrm{C}$.

\section{Melanoma xenograft model}

$200 \mu \mathrm{L}$ single-cell suspension of B16-F10 melanoma cells $\left(3 \times 10^{5}\right)$ was implanted subcutaneously into the left flank of mice. Tumor size (width $\times$ length; $\mathrm{mm}^{2}$ ) was determined with a caliper every 2 days. Tumor volume was calculated as previously described (16).

\section{HIFU treatment}

All experimental mice were randomly divided into shamHIFU and HIFU groups when the diameter of larger tumor reached 7 to $8 \mathrm{~mm}$. The HY2900 HIFU tumor therapy system (Haying Tech., Wuxi, China) was used for HIFU ablation in this study. The mice were anaesthetized using ketamine $(2 \mathrm{~mL} / \mathrm{kg})$ through intravenous injection. After anesthesia, the skin on top of the tumor nodule area was shaved and layed with ultrasound transmission gel. The mice of HIFU group were treated with $4.5 \mathrm{~W}$ and 9.2 $\mathrm{MHz}$ ultrasound. Treatment was performed point by point, and started at the center of the nodule with $6 \mathrm{~mm}$ therapy depth. Each point was treated for $1 \mathrm{~min}$, with $1 \mathrm{sec}$ pulse duration, and $5 \mathrm{sec}$ exposure separation. The procedures of sham-HIFU group were similar to HIFU group with no HIFU exposure.

\section{Pulmonary metastasis assay}

The mice bearing primary tumors were intravenously injected with B16-F10 cells at 7 days after HIFU treatment and monitored until death. The volume of melanoma tumor, number of pulmonary metastasis and cumulative survival rate were measured and recorded. The health mice without melanoma xenograft were used as the normal control group in this study.

\section{Histological analysis}

The tumor modules were excised from flank skin and fixed in $4 \%$ paraformaldehyde overnight and then paraffin embedding, sectioning, and hematoxylin eosin (H\&E) staining were performed as previously described (17).

\section{Enzyme-linked immunosorbent assay (ELISA)}

The blood was collected from normal, sham-HIFU and HIFU mice after 14 days of treatment, centrifuged at 5000 $\mathrm{g}$ for $6 \mathrm{~min}$. The plasma concentrations of IFN- $\gamma, \mathrm{TNF}-\alpha$, interleukin (IL)-6, and TGF- $\beta$ were determined by using the Beyotime Biotechnology (Shanghai, China) specific ELISA kits: mouse IFN $\gamma$ ELISA kit (PI508), mouse TNF- $\alpha$ ELISA kit (PT512), mouse IL-6 ELISA kit (PI326), and mouse/rat TGF- $\beta$ ELISA kit (PT878), following the 
PerkinElmer, Singapore). The cell viability of each well was normalized to that of the control. All cytotoxicity assays were conducted in triplicates (six wells per sample for each time point). Viability of the NSCs after treatments of various CM was determined using the MTT assay as well as LIVE/DEAD viability/cytotoxicity kit (Invitrogen, USA).

\section{Flow cytometry analysis}

The tumor nodules were placed individually in $0.1 \%$ collagenase type IV solution (Thermo Fisher Scientific, Waltham, USA), cut into small pieces and incubated at $37{ }^{\circ} \mathrm{C}$ for $30 \mathrm{~min}$. The digested tumor tissue was filtered through a $40 \mu \mathrm{m}$ cell strainer and then centrifuged at $500 \mathrm{~g}$ for $10 \mathrm{~min}$ at $4^{\circ} \mathrm{C}$. The pellet was washed twice and then resuspended to acquire single melanoma tumor cell suspension. Tregs were identified by Foxp3+ (\#565773-80, Invitrogen, Carlsbad, USA), CD4+ (\#100405, BioLegend, San Diego, USA) and CD45+ (\#147716, BioLegend, San Diego, USA). Th17 cells were identified by CD4+ and IL-17+ (\#506903, BioLegend, San Diego, USA). CD8+ tumor infiltrating $\mathrm{T}$ lymphocytes were identified by CD45+ and CD8-APC (\#100734, BioLegend, San Diego, USA).

\section{The microRNA (miRNA, miR) prediction}

The prediction of miRNAs which could bind to TGF- $\beta$ was performed by using TargetScan (http://www. targetscan.org/vert_72/) and ENCORI (http://starbase. sysu.edu.cn/) online package and database, following the instructions.

\section{Transfection and RNAi}

B16-F10 cells (60\% confluence) were transfected with 20 nM miR-9-5p, miR-9-5p negative control (NC), antimiRNA oligonucleotide (AMO)-miR-9-5p or negative control miRNA using the Lipofectamine 3000 reagent (Thermo Fisher Scientific, Waltham, USA), following the manufacturer's instruction. The cells were harvested $48 \mathrm{~h}$ after transfection for mRNA and protein analysis.

\section{Quantitative real-time polymerase chain reaction (qRT-PCR)}

Total RNA was prepared from melanoma tumor tissues or B16-F10 cells using the TRIzol kit (Invitrogen, Carlsbad, USA). $1 \mu \mathrm{g}$ total RNA was transcribed reversely into cDNA using the M-MLV Reverse Transcriptase (Thermo Fisher Scientific). The qRT-PCR was performed using the CFX Real-Time PCR Detection System (BioRad, Hercules, USA) with the SYBRTM Green PCR Master Mix (Thermo Fisher Scientific, Waltham, USA). Target gene expression level was normalized using the $2^{-\triangle \Delta \mathrm{CT}}$ method with GAPDH as the internal control. Primers for the RT-qPCR were: TGF- $\beta$, forward 5'-ACT GGA GTT GTA CGG CAG TG-3', reverse 5'-GGC TGA TCC CGT TGA TTT CC-3'; GAPDH, forward 5'-CGC TCT CTG CTC CTC CTG TT-3', reverse 5'-CCA TGG TGT CTG AGC GAT GT-3'.

\section{Immunoblotting analysis}

B 16-F10 cells or frozen melanoma tumor tissues were homogenized and lysed using the radioimmunoprecipitation assay buffer (Thermo Fisher Scientific, Waltham, USA). Samples were detected using Western blotting as described previously (18). The primary antibodies of TGF- $\beta$ (ab92486, 1:1000 dilution) and GAPDH (EPR16891, 1:2000 dilution) were ordered from Abcam (Cambridge, MA, USA).

\section{Luciferase reporter assay}

B16-F10 cells were cultured in 12-well plates at the density of $6 \times 10^{4}$ cells/per well $24 \mathrm{~h}$ before transfection. The TGF- $\beta$ 3'UTR-WT or TGF $\beta$ 3'UTR-mut reporter was co-transfected with pRLSV40 into the B16-F10 cells. Luciferase activity was measured by using the DualLuciferase Reporter Assay System (Promega, Madison, USA). The results were normalized by the value of Renilla activity and presented as fold change to the control group.

\section{Statistical analysis}

Statistical analysis was performed using Prism 8 software. Student's t test, one-way or two-way analysis of variance (ANOVA) were used to analyze the differences between groups. Data were shown as mean \pm standard deviation (SD).

\section{Results}

\section{HIFU exposure suppresses melanoma tumor progression}

The melanoma tumor bearing mice were administrated with sham-HIFU or HIFU, respectively, H\&E staining result showed that the cell proliferation of melanoma in HIFU exposure mice was slowed down compared to that in the sham group mice (Figure 1A). Notably, HIFU exposure significantly inhibited the primary tumor growth. 22 days after B16-F10 cell subcutaneous injection, tumor volume in mice of the sham-HIFU group were 2-4-fold larger than that in the HIFU-treated mice (Figure 1B). We further investigated the pulmonary metastasis in mice with primary tumors after B16-F10 cell intravenous injection, as shown in Figure 1C, HIFU exposure markedly reduced the number of tumor nodules in the lung compared to the sham-HIFU treatment (19 vs 34, $\mathrm{P}<0.01$ ). Moreover, the cumulative survival rate showed that HIFU exposure mice had much longer survival rate than mice in the sham control group (Figure 1D, $\mathrm{P}<0.05$ ). These results indicated that HIFU exposure displayed promising antitumor effects on inhibiting tumor growth, attenuating pulmonary metastasis and improving host survival in mouse melanoma model.

\section{HIFU exposure improves the anti-tumor immune response}

Immune response was detected in HIFU and sham-HIFU treated mice by measuring serum concentrations of tumor 
A

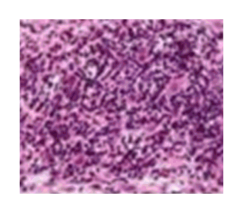

Sham-HIFU

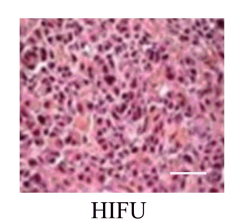

B

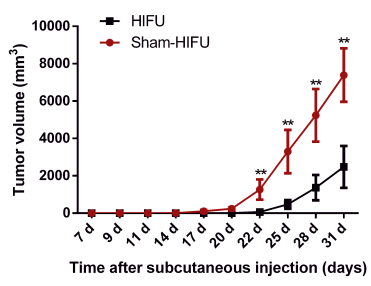

C
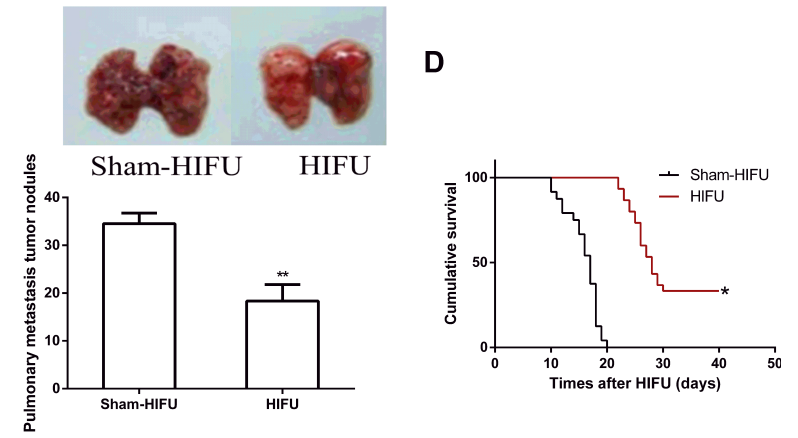

Figure 1. HIFU exposure inhibited melanoma growth and pulmonary metastasis. (A) H\&E staining of tumor tissues 11 days after subcutaneous injection (immediately after HIFU treatment) Scale bar, $20 \mu \mathrm{m}(\mathrm{n}=6)$. (B) The average tumor volumes of HIFU and sham-HIFU mice were plotted $(n=6)$. (C) Pulmonary metastasis tumor nodules were counted macroscopically after natural death $(n=10)$. (D) The cumulative survival rate was analyzed by log rank test $(n=30)$. ${ }^{*} P<0.05,{ }^{* *} P$ $<0.01$ compared to sham-HIFU mice.

microenvironment-associated cytokines, including IFN- $\gamma$, TNF- $\alpha$, IL- 6 and TGF- $\beta$. Pleiotropic molecule IFN- $\gamma$ has been reported to possess the anti-proliferative, proapoptotic and anti-tumor abilities (19). As shown in Figure $2 \mathbf{A}$, the concentration of IFN- $\gamma$ in the serum of HIFU exposure mice was 2-fold higher than that in the sham-HIFU mice (68.3 pg/mL vs $32.7 \mathrm{pg} / \mathrm{mL})$. Two proinflammatory cytokines, TNF- $\alpha$ and IL-6, were relatively higher in melanoma mice compared to normal mice. However, there was no difference between HIFU and sham-HIFU mice (Figure 2B and 2C). The regulatory cytokine TGF- $\beta$ is an important enforcer of immune tolerance, and tumors that secrete high levels of TGF- $\beta$ may escape from immune surveillance (20). Here, we found that the TGF- $\beta$ serum level was significantly decreased in HIFU treated mice compared to the sham control mice (Figure 2D). These data suggested the HIFU exposure might promote anti-cancer immunity via modulating cytokine secretion.

HIFU exposure inhibits Tregs and Th17 cells, and activates CD8+ tumor infiltrating lymphocytes (TILs)

To further investigate the anti-tumor immunity improvements after HIFU exposure, we detected the subset of CD4+ and CD8+ T cells using flow cytometric analysis. Tregs (Foxp3+CD4+/CD45+), the immunosuppressive subset of CD4+ T cells, was significantly increased in melanoma mice compared to normal control
(1.19\% vs $3.23 \%)$. Noticeably, in comparison with mice with sham-HIFU treatment, Treg population in HIFUtreated mice was significantly decreased (Figure 3). Th17 cells also increase tumor progression by activating angiogenesis and immunosuppressive activities (21). In line with the results of Tregs, the Th17 cell population (CD4+/IL-17+) in HIFU exposure mice was $2.49 \%$, which was markedly lower than that in sham-HIFU mice $(4.47 \%$,

Figure 4). CD8+ TILs have critical tumor suppressive roles. As shown in Figure 5, there was almost 3-fold increase in the $\mathrm{CD} 8+(\mathrm{CD} 45+/ \mathrm{CD} 8+)$ population in HIFU exposure mice compared to sham-HIFU mice $(17.59 \%$ vs $6.18 \%$ ). All these flow cytometric data indicated that HIFU exposure suppressed the immune tolerance and improved tumoricidal effector response in tumor tissues.

\section{HIFU exposure promotes anti-tumor immunity through} attenuating TGF- $\beta$ expression in melanoma tissues

Since TGF- $\beta$ plays a very important role in promoting the generation and differentiation of Tregs and Th17 subsets from naïve CD4+ T cells (22). We searched the candidate regulators of TGF- $\beta$ in StarBase/ENCORI database and identified miR-9-5p, which was inhibited in melanoma tumor tissues and restored by HIFU treatment (Figure 6A). Then we tested the TGF- $\beta$ expression in mouse melanoma samples with or without HIFU exposure. Both the mRNA and protein levels of TGF- $\beta$ were significantly increased in melanoma tissues, notably, the up-regulated TGF- $\beta$ expression was markedly inhibited by HIFU treatment (Figure 6B-6D). These results suggested that the antitumor effect of HIFU exposure might be mediated by the miR-9-5p/TGF- $\beta$ pathway.

\section{MiR-9-5p mediates HIFU-induced TGF- $\beta$ down- regulation in melanoma cells}

In order to investigate the mechanism of miR-9-5p-

A
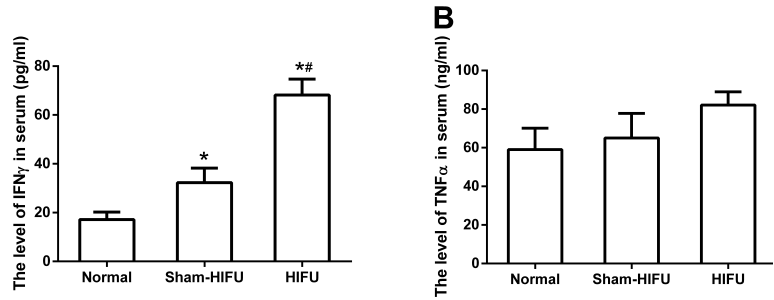

C
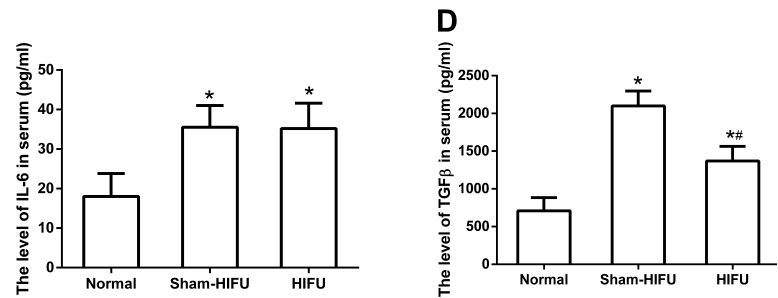

Figure 2. HIFU treatment enhanced antitumor immune response. IFN- $\gamma(A)$, TNF- $\alpha(B), I L-6(C)$ and TGF- $\beta$ (D) in mice serum were analyzed by ELISA. ${ }^{*} \mathrm{P}<0.05$ as compared with normal, $\# \mathrm{P}<0.05$ as compared with sham-HIFU $(n=6)$. 


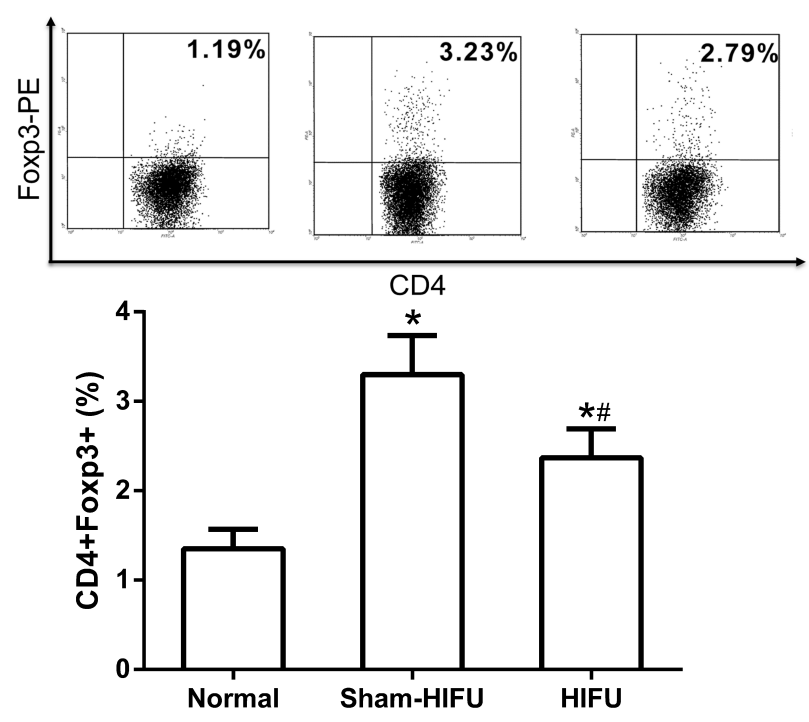

Figure 3. Flow cytometric analysis of Foxp3+CD4+/CD45+ Tregs in indicated groups (14 days after HIFU exposure). ${ }^{*} \mathrm{P}$ $<0.05$ as compared with normal, \#P $<0.05$ as compared with sham-HIFU $(n=6)$.

mediated TGF- $\beta$ regulation in melanoma, we performed miR-9-5p gain-of-function, loss-of-function, and promoter binding tests using RNAi and dual-luciferase reporter assay in B16-F10 cells. As shown in Figure 7A, in comparison with the control group, miR-9-5p overexpression could significantly reduce the mRNA level of TGF- $\beta$ in B16-F10 cells. In contrast, the TGF- $\beta$ expression level was dramatically increased when miR9-5p was blocked by AMO-miR-9-5p (Figure 7A). The same regulatory pattern was observed at the translational level, where miR-9-5p gain-of-function decreased TGF- $\beta$ protein level, while miR-9-5p loss-of-function significantly increased TGF- $\beta$ protein level (Figure 7B and $7 \mathrm{C}$ ). The sequence analysis result showed that miR$9-5 p$ was highly complementary with the TGF- $\beta 3$ 'UTR (Figure 7D). To further investigate whether miR-9-5p could directly bind to TGF- $\beta$ promoter, we constructed the luciferase reporter using TGF- $\beta$ 3'UTR-wt region and mutated 3'UTR sequence (TGF- $\beta$ 3'UTR-mut). We found that miR-9-5p could significantly reduce the luciferase activity derived by TGF- $\beta$ 3'UTR-wt instead of TGF- $\beta$ 3'UTR-mut. These data indicated that TGF- $\beta$ was a target of miR-9-5p that mediated the HIFU-induced TGF- $\beta$ down-regulation, and then promoted anti-tumor immunity in melanoma.

\section{Discussion}

HIFU is a promising method for the non-invasive ablation of various tumors $(6,13)$. In this study, we applied the HIFU technique for melanoma treatment and demonstrated that HIFU exposure suppressed the proliferation and growth of the primary tumors, inhibited pulmonary metastasis, and improved the cumulative survival rate of melanoma mice. HIFU treatment not
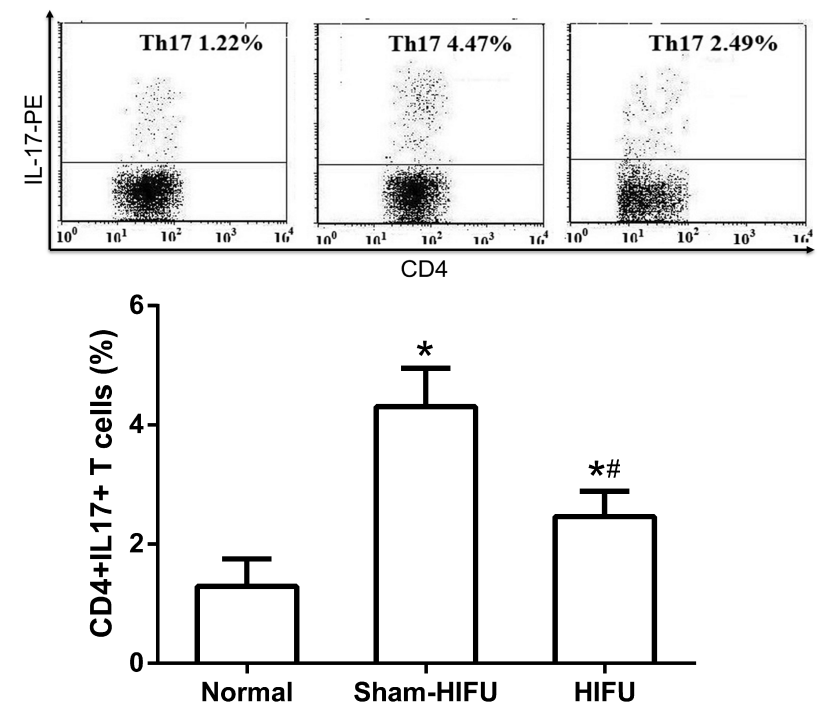

Figure 4. Flow cytometric analysis of CD4+/IL-17+ Th17 cells in indicated groups (14 days after HIFU exposure). ${ }^{*} P<0.05$ as compared with normal, \#P $<0.05$ as compared with shamHIFU $(n=6)$.

only activates immune response including IFN- $\gamma$ upregulation and TGF- $\beta$ down-regulation but also changes $\mathrm{T}$ cell subsets in tumor microenvironments, such as suppression of Tregs and Th17 cells and activation of CD8+ effector T cells. In line with our findings, Liu et al. reported that HIFU treatment significantly stimulated DC maturation and tumor infiltration surrounding the thermal lesion compared to the control in the MC-38 and B16 tumor models (23). Moreover, the enhanced cytotoxic $\mathrm{T}$ lymphocyte activity and elevated IFN- $\gamma$ secretion accompanying the expansion of DCs in lymph nodes were observed by another research group who performed HIFU
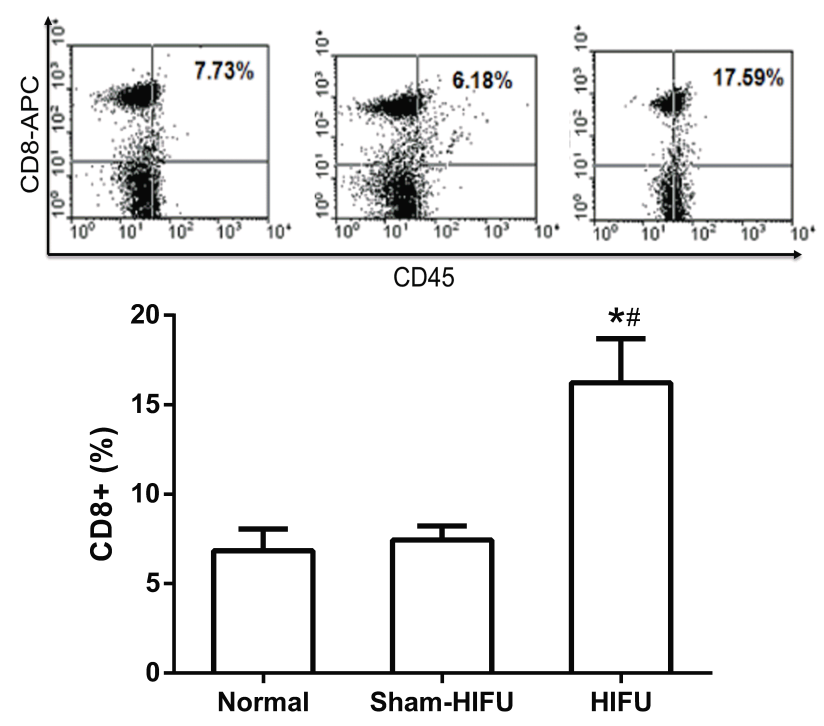

Figure 5. Flow cytometric analysis of CD8+ TILs in indicated groups (14 days after HIFU exposure). ${ }^{*} P<0.05$ as compared with normal, \#P $<0.05$ as compared with sham-HIFU $(n=6)$. 
A

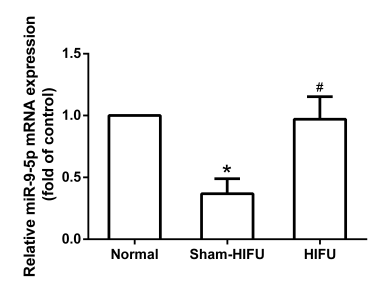

C

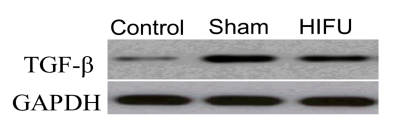

B

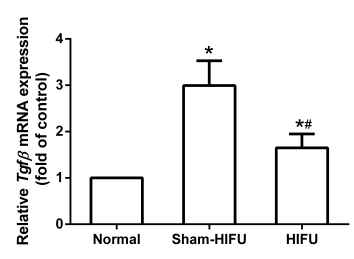

D

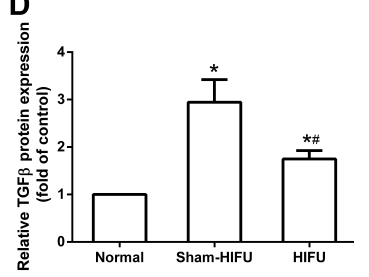

Figure 6. HIFU treatment caused the differential expressions of miR-9-5p and TGF- $\beta$. A-B. The levels of miR-9-5p (A) and TGF- $\beta$ (B) in tumor tissues were detected by qPCR. (C-D) The levels of protein of TGF- $\beta$ in tumor tissues were detected by Western blot. ${ }^{*} P<0.05$ as compared with normal, \#P $<0.05$ as compared with sham-HIFU $(n=6)$.

treatment on colon adenocarcinoma tumors (12). Based on the above findings, the activation of anti-tumor immunity might be one of the major advantages of HIFU treatment.

The $\mathrm{T}$ cell subset alteration after HIFU exposure is another important beneficial effect of HIFU treatment. CD8+ T cells are able to differentiate into cytotoxic $\mathrm{T}$ lymphocytes upon the activation of antigen-presenting cells, which then exert an efficient anti-tumor attack (24). However, Tregs play essential roles in maintaining the immune system self-tolerance and immunosuppression, thus promote tumor development and progression (25). In addition, Th17 cells are a double-edged sword in tumor immunity: on the one hand Th17 cells promote tumor progression through increasing angiogenesis and immunosuppression, while on the other hand Th17 cells induce the anti-tumor immune response by improving effector $\mathrm{T}$ cell filtration and IFN- $\gamma$ secretion (22). In the current study, we found that HIFU exposure significantly reduced the population of Tregs and Th17 cells, while increased CD8+ effector $\mathrm{T}$ cells in melanoma tumor tissues. Meanwhile, alteration in other subsets of immune cells has been observed by previous studies. Fifteen pancreatic cancer patients were treated by HIFU exposure in a clinical trial, and the authors found the CD4+, CD4+/CD8+ and natural killer (NK) cells were increased in the serum of 10 patients, and NK cell activity was dramatically increased after HIFU treatment (26). Similarly, in a HCC cell vaccine study, the researchers found the cytotoxicity of cytotoxic $\mathrm{T}$ lymphocytes was highly increased after HIFU exposure, and these vaccines displayed stronger anti-tumor ability compared to non-HIFU ones (27). All these results suggest that HIFU treatment could induce systemic change in the tumor microenvironment, which consequently improves anti-tumor immunity.

MiRNAs play important roles in T lymphocyte

differentiation and maturation, as well as immune response and immune tolerance (28). TGF- $\beta$ promotes the CD4+ derived Treg differentiation, which mediates immunosuppression in various tumors $(25,29)$. Here, we have found that TGF- $\beta$ is a target of miR-9-5p, the expression of which is significantly up-regulated by HIFU exposure in melanoma tissues. Luciferase reporter assay has confirmed that miR-9-5p is able to bind to TGF- $\beta$ 3'UTR directly. Overexpression of miR-9-5p could inhibit TGF- $\beta$ expression on both mRNA and protein levels in melanoma cells. A recent study showed that miR134 targeted CD86, a T lymphocyte activation antigen, in mouse melanoma cells. HIFU exposure dramatically inhibited miR-134, then enhanced CD86 expression, which could promote the secretion of IFN- $\gamma$ and TNF- $\alpha$, and improve anti-tumor immunity in the melanoma allograft mouse model (16). In line with these results, Li et al. examined the therapeutic effect of HIFU on colorectal cancer metastasis and found that HIFU exposure increased the expression of miR-124, which targeted STAT3, a transcription factor overexpressed and activated in various cancer cells and tumor-associated immune cells, and then inhibited invasion and metastasis of colon cancer cells (30). The in vitro regulation of miR-9-5p/TGF- $\beta$ pathway in melanoma cells has been demonstrated by this study, but whether they also play key regulatory roles in antitumor immunity in vivo is still unknown. Future work will further validate the in vivo regulation of HIFU-mediated miR-9-5p/TGF- $\beta$ pathway in melanoma treatment.

In the current study, we have demonstrated that HIFU
A

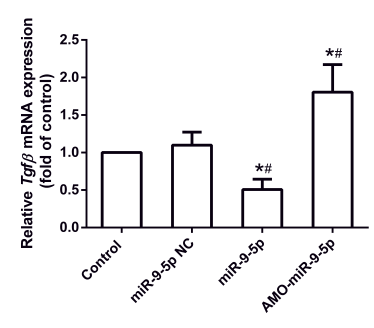

C

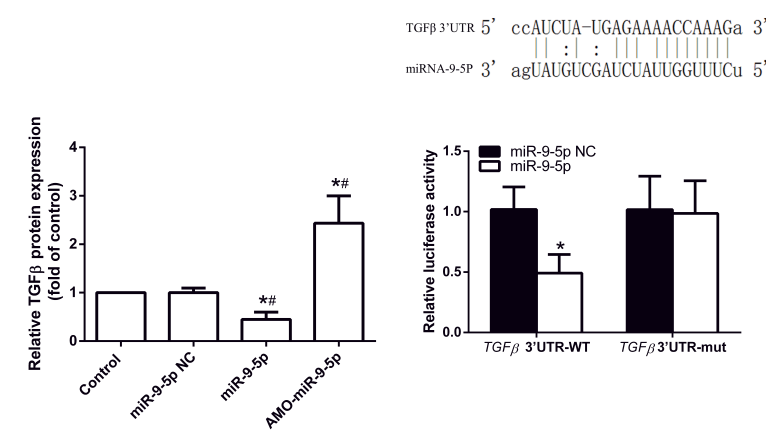

Figure 7. TGF- $\beta$ was a target of miR-9-5p in melanoma cells. Delivery of miRNA-9-5p significantly decreased the TGF- $\beta$ mRNA (A) and protein (B and C) expression levels in B16-F10 cells. (D) MiR-9-5p targeting TGF- $\beta$ was validated by dual-luciferase reporter assay. ${ }^{*} \mathrm{P}<0.05$ vs control, \#P<0.05 vs miR-9-5p-NC $(n=6)$. 
exposure could suppress melanoma growth and metastasis in mouse and cell models. HIFU treatment activates antitumor immune response, inhibits Tregs and Th17 cells, and increases CD8+ effector T cells in melanoma. We also reveal that TGF- $\beta$ is a direct target of miR-9-5p. Mechanistically, the anti-tumor effect of HIFU might be mediated by up-regulating miR-9-5p and then suppressing the expression of TGF- $\beta$ in melanoma cells.

\section{Conflict of interest}

The authors declare that they have no conflicts of interest to disclose.

\section{Funding}

This work was supported by National Natural Science Foundation of China (81703751).

\section{References}

1 Miller AJ, Mihm MC, Jr. Melanoma. N Engl J Med. 2006;355:51-65.

2 Schadendorf D, van Akkooi ACJ, Berking C, Griewank KG, Gutzmer R, Hauschild A, et al. Melanoma. Lancet. 2018;392:971-984.

3 Zbytek B, Carlson JA, Granese J, Ross J, Mihm MC, Jr., Slominski A. Current concepts of metastasis in melanoma. Expert Rev Dermatol. 2008;3:569-585.

4 Dadras SS, Paul T, Bertoncini J, Brown LF, Muzikansky A, Jackson DG, et al. Tumor lymphangiogenesis: a novel prognostic indicator for cutaneous melanoma metastasis and survival. Am J Pathol. 2003;162:1951-1960.

5 Hsiao YH, Kuo SJ, Tsai HD, Chou MC, Yeh GP. Clinical application of high-intensity focused ultrasound in cancer therapy. J Cancer. 2016;7:225-231.

6 Maloney E, Hwang JH. Emerging HIFU applications in cancer therapy. Int J Hyperthermia. 2015;31:302-309.

7 Xiong LL, Hwang JH, Huang XB, Yao SS, He CJ, Ge XH, et al. Early clinical experience using high intensity focused ultrasound for palliation of inoperable pancreatic cancer. JOP. 2009;10:123-129.

8 Ritchie RW, Leslie T, Phillips R, Wu F, Illing R, ter Haar G, et al. Extracorporeal high intensity focused ultrasound for renal tumours: a 3-year follow-up. BJU Int. 2010;106:1004-1009.

$9 \mathrm{Ng} \mathrm{KK}$, Poon RT, Chan SC, Chok KS, Cheung TT, Tung $\mathrm{H}$, et al. High-intensity focused ultrasound for hepatocellular carcinoma: a single-center experience. Ann Surg. 2011;253:981-987.

10 Li S, Wu PH. Magnetic resonance image-guided versus ultrasound-guided high-intensity focused ultrasound in the treatment of breast cancer. Chin J Cancer. 2013;32:441452.

11 Uchida T, Tomonaga T, Kim H, Nakano M, Shoji S, Nagata $Y$, et al. Improved outcomes with advancements in high intensity focused ultrasound devices for the treatment of localized prostate cancer. J Urol. 2015;193:103-110.

12 Hu Z, Yang XY, Liu Y, Sankin GN, Pua EC, Morse MA, et al. Investigation of HIFU-induced anti-tumor immunity in a murine tumor model. J Transl Med. 2007;5:34.

13 Wu F, Zhou L, Chen WR. Host antitumour immune responses to HIFU ablation. Int $\mathrm{J}$ Hyperthermia. 2007;23:165-171.
14 Wang YN, Khokhlova T, Bailey M, Hwang JH, Khokhlova V. Histological and biochemical analysis of mechanical and thermal bioeffects in boiling histotripsy lesions induced by high intensity focused ultrasound. Ultrasound Med Biol. 2013;39:424-438.

15 Xia JZ, Xie FL, Ran LF, Xie XP, Fan YM, Wu F. High-intensity focused ultrasound tumor ablation activates autologous tumor-specific cytotoxic T lymphocytes. Ultrasound Med Biol. 2012;38:1363-1371.

16 Yuan SM, Li H, Yang $M$, Zha $H$, Sun $H$, Li XR, et al. High intensity focused ultrasound enhances anti-tumor immunity by inhibiting the negative regulatory effect of miR134 on CD86 in a murine melanoma model. Oncotarget. 2015;6:37626-37637.

17 Fischer AH, Jacobson KA, Rose J, Zeller R. Hematoxylin and eosin staining of tissue and cell sections. CSH Protoc. 2008;2008:pdb prot4986.

18 Guo L, Zhang P, Chen Z, Xia H, Li S, Zhang Y, et al. Hepatic neuregulin 4 signaling defines an endocrine checkpoint for steatosis-to-NASH progression. J Clin Invest. 2017;127:4449-4461.

19 Doherty GM, Lange JR, Langstein HN, Alexander HR, Buresh CM, Norton JA. Evidence for IFN-gamma as a mediator of the lethality of endotoxin and tumor necrosis factor-alpha. J Immunol. 1992;149:1666-1670.

20 Detournay O, Schnitzler CE, Poole A, Weis VM. Regulation of cnidarian-dinoflagellate mutualisms: Evidence that activation of a host TGFbeta innate immune pathway promotes tolerance of the symbiont. Dev Comp Immunol. 2012;38:525-537.

21 Chalmin F, Mignot G, Bruchard M, Chevriaux A, Vegran F, Hichami A, et al. Stat3 and Gfi-1 transcription factors control Th17 cell immunosuppressive activity via the regulation of ectonucleotidase expression. Immunity. 2012;36:362-373.

22 Knochelmann HM, Dwyer CJ, Bailey SR, Amaya SM, Elston DM, Mazza-McCrann JM, et al. When worlds collide: Th17 and Treg cells in cancer and autoimmunity. Cell Mol Immunol. 2018;15:458-469.

23 Liu F, Hu Z, Qiu L, Hui C, Li C, Zhong P, et al. Boosting highintensity focused ultrasound-induced anti-tumor immunity using a sparse-scan strategy that can more effectively promote dendritic cell maturation. J Transl Med. 2010;8:7.

24 Gonzalez H, Hagerling C, Werb Z. Roles of the immune system in cancer: from tumor initiation to metastatic progression. Genes Dev. 2018;32:1267-1284.

25 Togashi Y, Shitara K, Nishikawa H. Regulatory T cells in cancer immunosuppression - implications for anticancer therapy. Nat Rev Clin Oncol. 2019;16:356-371.

26 Wang $X$, Sun J. High-intensity focused ultrasound in patients with late-stage pancreatic carcinoma. Chin Med J (Engl). 2002;115:1332-1335.

27 Zhang Y, Deng J, Feng J, Wu F. Enhancement of antitumor vaccine in ablated hepatocellular carcinoma by high-intensity focused ultrasound. World J Gastroenterol. 2010;16:35843591.

28 Lee HM, Nguyen DT, Lu LF. Progress and challenge of microRNA research in immunity. Front Genet. 2014;5:178.

29 Keswani T, Sarkar S, Sengupta A, Bhattacharyya A. Role of TGF-beta and IL-6 in dendritic cells, Treg and Th17 mediated immune response during experimental cerebral malaria. Cytokine. 2016;88:154-166.

30 Li M, Wan G, Yu H, Xiong W. High-intensity focused ultrasound inhibits invasion and metastasis of colon cancer cells by enhancing microRNA-124-mediated suppression of STAT3. FEBS Open Bio. 2019;9:1128-1136. 